\title{
An External Secondary Ion Source for Fourier Transform Mass Spectrometry
}

\author{
Carl F. Ijames and Charles L. Wilkins \\ Department of Chemistry, University of California, Riverside, California, USA
}

A differentially pumped external secondary ion source for Fourier transform mass spectrometry is described. Installation does not interfere with other experiments such as laser desorption or photodissociation. Spectra of cesium iodide clusters (with ions up to $\mathrm{m} / z$ 8187), polyethylene glycol 1000, and histidine from both glycerol and dithiothreitol-dithioerythritol matrices are reported. The ion with $\mathrm{m} / \mathrm{z} 156$ from histidine was recorded with mass resolution of 160,000. (J Am Soc Mass Spectrom 1990, 1, 208-216)

$\mathrm{F}$ ourier transform mass spectrometry (FTMS) is a versatile and powerful method of performing mass analysis. Its demonstrated ultrahigh mass resolution and mass range offer great promise for the analysis of high-mass biomolecules [1]. The most widely used ionization technique for thermally labile and/or nonvolatile materials with molecular masses up to $10,000 \mathrm{u}$ is liquid secondary ion mass spectrometry (LSIMS) or fast atom bombardment (FAB) [2]. Coupling LSIMS (or FAB) with FTMS would allow the analysis of many compounds not readily amenable to laser desorption, the most common soft ionization technique for FTMS.

The major difficulty in interfacing LSIMS with FTMS is the volatile matrix. Glycerol, the most common matrix, has a room-temperature vapor pressure of approximately $1 \times 10^{-5}$ torr. Resolution in FTMS is inversely proportional to the pressure in the trappedion cell, and operating pressures less than $5 \times 10^{-9}$ torr are typical when maximum resolution is desired. A second consideration is that the most common LSIMS primary beams consist of neutral atoms, typically argon or xenon, formed by discharge ionization followed by resonant charge exchange in a gas collision cell. This arrangement produces pressures in the $10^{-6}$ torr range, which is also too high for maximum resolution in FTMS. However, Aberth and Burlingame [3] demonstrated that the charge of the primary beam is unimportant when matrices that are at least moderately electrically conducting are employed, and they described a simple cesium ion gun that produces negligible gas load.

The most elegant solution to the incompatibilities of LSIMS and FTMS is to physically separate the ion generation and mass analysis regions. This technique was pioneered by McIver and co-workers, who formed ions in a standard differentially pumped quadrupole mass spectrometer source and guided them with a radio-

Address reprint requests to Professor Charles L. Wilkins, Department of Chemistry, University of California-Riverside, Riverside, CA 92521. frequency-only quadrupole through the fringing field of a 7-T superconducting magnet to an FTMS cell, where they were trapped and detected $[4,5]$. Hunt and co-workers [6] duplicated this design, and, with the addition of a cesium ion gun to perform LSIMS, they extended the demonstrated FTMS mass range for organic biomolecules to over $12,000 \mathrm{u}$.

Kofel and co-workers $[7,8]$ showed that the quadrupole ion guide was not mandatory by constructing a system of electrostatic lenses to focus ions formed by electron impact into a 4.7-T superconducting magnet. This approach was also followed by Smalley and co-workers [9] for the analysis of ions formed by photoionization in a supersonic expansion. Most recently, McIver and co-workers [10] constructed a second quadrupole-FTMS system operating at a magnetic field strength of $6 \mathrm{~T}$ and demonstrated impressive resolution at high mass (a resolution of 53,000 at $\mathrm{m} / z$ 9745).

\section{SIMS/FTMS}

The first SIMS/FTMS experiments were performed by desorbing ions immediately adjacent to the trappedion cell without a matrix. The primary beam was cesium ions formed using a gun similar to that described by Aberth and Burlingame [3]. This avoided the gas load of the neutral gun but constrained the primary beam to be along the magnetic field lines. Two arrangements of an on-axis primary beam are possible in FTMS. In the first, the ion gun replaces the normal electron gun. The primary beam traverses the cell and strikes the sample. Secondary ions are emitted back toward the cell, where they are trapped. This prevents other experiments from being performed while the gun is installed and requires alignment of the beam with holes in each trap plate. If the beam strikes a plate, secondary electrons and scattered primary ions can perturb the ion population in the cell. The second arrangement places the gun behind the sample, which is mounted on a partially transparent screen. Provision must still be made for the fraction of the primary beam 
that penetrates the sample to traverse the cell, but the resulting flux is much lower. The sample must now be inserted between the gun and the cell while both are on the central axis of the system. One method of accomplishing this is to mount a miniature gun on the end of a probe along with the sample holder.

Castro and Russell [11] obtained spectra using both approaches. They replaced the electron gun with a cesium ion gun and mounted the sample either on a screen on the first trap plate or on the rear trap plate itself. This necessitated cell removal to change samples but allowed them to acquire spectra of vitamin $B_{12}$ [11]. The second approach, with the sample supported on a probe, was also followed by Amster et al. [12], who reported the observation of cesium iodide clusters with $m / z$ up to 16,241 .

\section{Magnetron Emission}

In the present work, during development of a probemounted cesium gun, it was discovered that some time after insertion of the gun into the vacuum system and application of high voltage $(3-6 \mathrm{kV})$, the gun began transmitting radiation at frequencies between $10^{5}$ and $10^{7} \mathrm{~Hz}$. The initial intensity was quite low and appeared as noise in the mass spectrum, but eventually the intensity increased to the point where computer operation was disrupted. The time before emission began was a direct function of the pressure and the voltage, but even at $3 \mathrm{kV}$ and $3 \times 10^{-9}$ torr, radiation was detectable within several minutes. The problem persisted even when the probe was withdrawn, so that the magnetic field at the gun was reduced from $3 \mathrm{~T}$ to approximately $10^{-2} \mathrm{~T}$, although the frequency of the radiation did decrease. Investigation of various electrical feedthrough and gun electrode designs revealed that the problem was exacerbated by high electric fields (close electrode spacing) and cylindrical symmetry of the electrodes along the magnetic field.

The explanation of this radiated noise lies in the observation that this geometry is the same as that found in a magnetron tube. Consider an electron produced by cold cathode emission from the grounded periphery around the central high-voltage conductor in a feedthrough. It is accelerated toward the central conductor, but the magnet curves its path into a circular arc, and so it is returned to the surface near where it was emitted. However, if it collides with a gas molecule during its flight, electron ionization (EI) can occur and produce a second electron and a positive ion. If these electrons are scattered far enough from the surface, they can establish stable orbits around the central conductor and eventually undergo further collisions to produce still more electrons. The motion of the electron during this orbit is termed magnetron motion, and the time required by the electron to proceed once around the central conductor defines the magnetron frequency. This frequency is determined by the strength of the electric and magnetic fields and ranges from $10^{3}$ to $10^{8} \mathrm{~Hz}$ under these conditions. The positive ion will be accelerated away from the central conductor and may establish a stable orbit but most likely will be lost. The crossed electric and magnetic fields thus provide both a trapping mechanism for electrons in the $x y$ plane and a production mechanism that causes the electron population to grow exponentially until limited either by space-charge modification of the electric field or losses along the magnetic field.

Electrons that drift along the magnetic field and collide with the insulating surface of the feedthrough cause electrostatic charging of this surface. The potential falls until it equals the kinetic energy of the approaching electrons and so reflects any additional electrons that approach. In the direction away from the feedthrough, the converging magnetic field lines form a magnetic mirror that reflects some fraction of the electrons. Also, any insulating surfaces used to support the conductor or the ion gun become charged and prevent loss in this direction. Thus, a complete trap is formed, and the electron population rises until limited by space charge. The trapped electrons proceed along the magnetron orbits at their characteristic frequencies, and this moving cloud of charge induces currents in nearby conductors, which act as antennas to broadcast the signal. Circular symmetry serves to enhance the quality of the trap and accelerate the accumulation of electrons, and high pressures increase the rate of ion formation. *

This process effectively prevents the placement of high-voltage feedthroughs on-axis in an FTMS, unless the high voltage can be applied in a pulsed manner for times short compared to the onset of the radiated noise. For this reason most investigators abandoned the simple, on-axis approach to SIMS/FTMS.

\section{External Source Design}

In light of these considerations, it was decided to construct an external, differentially pumped LSIMS source. Further, it was intended that this source should not interfere with any other experiments, such as laser desorption or photodissociation, which require onaxis access to the cell. This dictated that the primary beam be transverse to the magnetic field and the gun mounted sufficiently far from the axis that it would not restrict access. Differential pumping would be provided by using a commercial differentially pumped dual-cell FTMS instrument. The secondary ions are created in the source region but outside the source cell and are transferred either into the source cell or on through the conductance limit to the low-pressure analyzer cell for analysis.

Kofel and co-workers [8] observed that the angular cone of acceptance into the magnetic mirror formed

\footnotetext{
* This explanation resulted in part from discussions with Sahba Ghaderi, Nicolet Analytical Instruments, Madison, Wisconsin, and Professor Dave H. Russell, Texas A\&M, College Station, Texas.
} 



Figure 1. SIMION simulation of the Antek cesium ion gun operated (a) with no magnetic field and (b) transverse to $0.165 \mathrm{~T}$. Emitter, extractor, focus, and exit potentials were $6,5.78,5$, and $0, \mathrm{kV}$, respectively.

by the fringing fields of the solenoidal magnet is determined by the ratio of the initial and final magnetic fields (eqs 1 and 2, ref. 8). The smaller this ratio (the further inside the magnet the ions are formed), the larger the acceptance angle and the lower the fraction of velocity along the $z$ axis that is converted into cyclotron motion for a given divergence angle of the incoming beam. Thus, there are advantages to placing the source at the highest possible magnetic field that still allows the primary ion gun to function.

The most convenient location at which to mount an ion gun perpendicular to the $z$ axis in a Nicolet FTMS-2000 dual cell instrument is at the source pumping cross. With a 3-T magnet, the field at that location is $0.165 \mathrm{~T}$, oriented along the $z$ axis. A field map showed that over the volume of the gun and sample holder the radial variation in field was less than $5 \%$, which was considered negligible. Figure 1 shows simulations of the ion paths out of the cesium ion gun both in the absence of magnetic field and transverse to a 0.165 -T field. As can be seen, some perturbation of the beam occurs, but a focused beam is still obtained. For comparison, simulations for a field of a 0.44 $\mathrm{T}$, which corresponds to the same location next to a 7.2-T magnet, were also performed (Figure 2). Now the deflection is so strong that no ions escape the gun. However, a combination of operating voltages that did

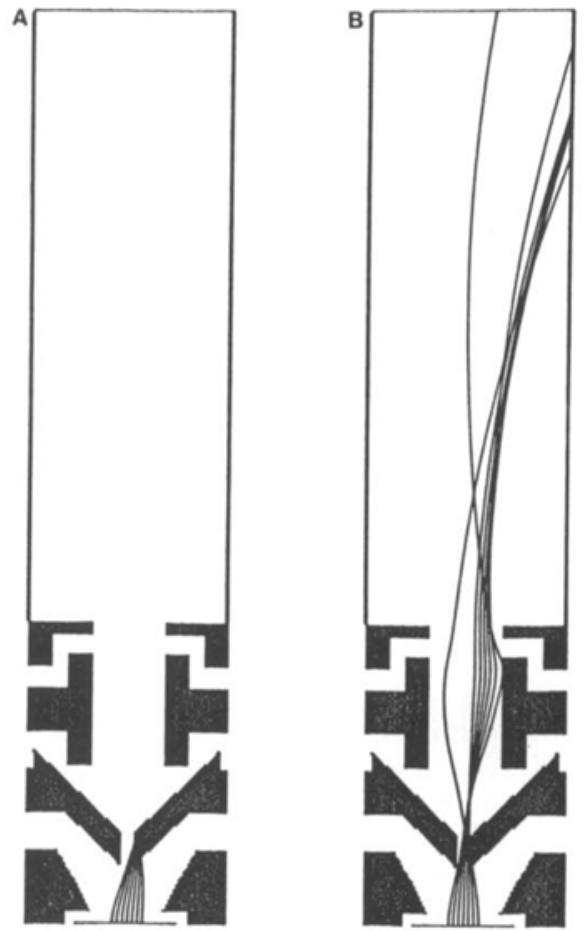

Figure 2. SIMION simulation of the Antek cesium ion gun operated transverse to $0.44 \mathrm{~T}$ (a) with normal potentials and (b) with an extractor potential of $0 \mathrm{~V}$.

produce a very weak, divergent output (see Figure $2 b$ ) was found. Each set of operating conditions was experimentally verified. The gun operates normally in the 3-T system, but no output current is obtained in the 7.2-T system. When the nonstandard potentials were used, there was a beam approximately 100 times less intense than the one produced by the same gun with the same power settings on the 3-T system. These potentials were provided by static power supplies, so the beam could not be pulsed on and off. After this, further development was pursued on the 3-T system.

\section{Experimental}

The external source is diagramed in Figure 3. The mass spectrometer is an FTMS-1000 (Nicolet Analytical Instruments, Madison, WI) upgraded with a standard FTMS-2000 dual cell ( $4.76 \mathrm{~cm}$ cubic cells) and chamber assembly and manual direct insertion probe. Diffusion pumps, with pumping speeds of 700 and 300 liters/s, respectively, were utilized on the source and analyzer and produced base pressures of $1 \times 10^{-8}$ and $6 \times 10^{-9}$ torr. The cesium gun (constructed by Antek, Inc., Palo Alto, CA) was mounted approximately $1 \mathrm{~cm}$ off center on a rotatable 2.75 -in. conflat flange such that the exit aperture was $0.635 \mathrm{~cm}$ from the cell support rods and $4.78 \mathrm{~cm}$ from the sample. The sample was placed on a $0.635 \mathrm{~cm}$ diameter tip angled at $45^{\circ}$ to the incident 


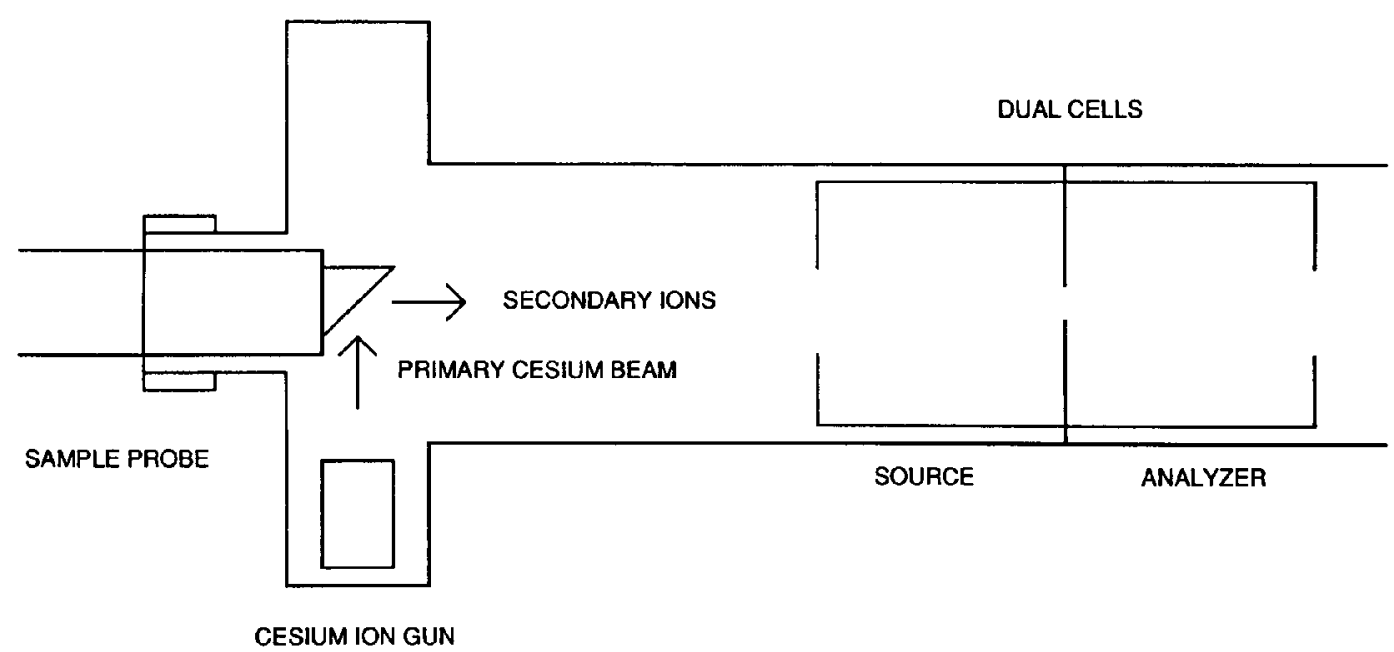

Figure 3. The external LSIMS/FTMS system.

beam. This tip was attached to the end of the manual direct insertion probe and inserted until the cesium beam struck the center, approximately $38 \mathrm{~cm}$ from the source trap plate. Vertical alignment was performed by rotating the flange that supported the gun, and horizontal alignment consisted of controlling the insertion depth of the probe to scan the beam across the angled surface.

A power supply constructed by Nicolet Analytical Instruments provided the heater current and the extraction, focus, and acceleration potentials for the cesium gun. The spot size was measured by recording the current as the probe was gradually inserted and by using an off-axis wire on the probe end and rotating the probe to intercept the beam. The focus voltage was optimized at 0.84 times the full acceleration voltage to minimize the spot size. The primary beam was pulsed on for 4-6 ms by switching the potential of the extractor between $50 \mathrm{~V}$ above the emitter potential and $200 \mathrm{~V}$ below the emitter potential. Typical operating potentials for the emitter, extractor, focus, and exit, respectively, were $6,5.78,5$, and $0 \mathrm{kV}$. Current measurements were made either with a Model 480 picoammeter (Keithley Instruments, Cleveland, $\mathrm{OH}$ ) or the electrometer section of an ion gauge controller (Model RG-31X, Veeco Instruments, Plainview, NY). The trap potential was $2 \mathrm{~V}$, and a 3-ms delay was inserted after the beam and before the excitation. The quench event before ionization was set to $100 \mathrm{~ms}$ to allow stabilization of the ion gun current between pulses.

Ion transfer efficiencies between the cells were determined by ratioing the abundance detected in the analyzer cell after transfer and the abundance detected in the source cell without transfer. No direct measurement of the total number of secondary ions produced at the sample or trapped in the cells was made.

Finally, some experiments were performed with the cesium gun mounted on the custom-built 7.2-T FTMS2000 and controlled by the Nicolet power supply and a
30-kV adjustable power supply (HiPotronics, Brewster, NY) used to provide variable extraction potentials.

All samples were purchased commercially and used as received. The cesium gun operation was modeled using the SIMION version 4.0 program operating on an IBM PC/AT-compatible computer.*

\section{Results and Discussion}

Initial experiments were performed to align and characterize the cesium ion gun. The beam was vertically aligned by venting the chamber and rotating the mounting flange that supported the gun. After the initial alignment, no further adjustments were necessary over the next several months. The gun produces a beam focused to a $2-\mathrm{mm}$ spot, with energy adjustable from several hundred electronvolts to $8.5 \mathrm{keV}$ and currents up to $2 \mu \mathrm{A}$. No degradation of output current has been observed after approximately $200 \mathrm{~h}$ of operation at the 500-nA level. When necessary, emitter replacement can be accomplished easily. The pulse width is limited by the optoisolator circuit in the power supply to a minimum width of $3 \mathrm{~ms}$, and the beam energy is constrained by the feedthroughs on the conflat flange.

The first sample to be examined was cesium iodide, with no matrix. The ions with peaks at $m / z 133$ and 393 were ejected to enhance the dynamic range of the higher mass ions. A portion of the spectrum is shown in Figure 4 . The cluster for $\mathrm{Cs}_{32} \mathrm{I}_{31}{ }^{+}, \mathrm{m} / \mathrm{z}$ 8187 , is clearly visible. Two higher mass peaks $(\mathrm{m} / \mathrm{z}$ 8966 and 9745 ) are also possibly present but with very marginal signal-to-noise ratio. The clusters were emitted from the surface with several electronvolts of kinetic energy, as potentials of -2 to $-6 \mathrm{~V}$ on the sample were necessary to deaccelerate the ions so that they could be trapped in the source cell. Transfer through

\footnotetext{
* SIMION is available from Dave Dahl, Idaho National Engineering Laboratory, EG\&G Idaho, Inc., Idaho Falls, Idaho.
} 




Figure 4. SIMS/FTMS spectrum of CSI clusters, recorded in the source cell.

the conductance limit was very inefficient (less than $1 \%$ ), probably because of the large spatial distribution of the ions in the source cell as a consequence of their large initial energy. The ionization beam duration was $5 \mathrm{~ms}$, and 15,400 scans were co-added. The receive time was $0.41 \mathrm{~s}$ per scan, for a total ionization time of $77 \mathrm{~s}$ and a total experiment time of $2.2 \mathrm{~h}$. Because the signal damped to the noise level after $0.1 \mathrm{~s}$, only the first fourth of the averaged transient was Fourier transformed, so the total time could have been reduced to $56 \mathrm{~min}$. The ionization time of $77 \mathrm{~s}$ compares very favorably with the time required to complete a single scan over the same mass range, $m / z 560-10,000$, on a conventional double-focusing mass spectrometer.

The next sample was polyethylene glycol 1000 , deposited as a solid film from a $\mathrm{KBr}$-methanol solution. The ionization time was $4 \mathrm{~ms}$ per scan, and 916 scans were co-added for a total ionization time of $3.7 \mathrm{~s}$. A molecular ion distribution consisting of $[\mathrm{M}+\mathrm{K}]^{+}$ions is observed, along with fragments whose abundance is approximately half that of the parent ions (see Figure 5). This represents much greater fragmentation than is usually observed with laser desorption/FTMS. The probe bias potential was $-1 \mathrm{~V}$, and ions could be transferred to the analyzer cell with approximately $10 \%$ efficiency.

Next, glycerol was inserted as a sample. With $10 \mu \mathrm{L}$ of glycerol on the probe tip, pressures remain approximately $4 \times 10^{-6}$ torr in the source and $2 \times 10^{-8}$ torr in the analyzer for $10 \mathrm{~min}$ before the glycerol evaporates. The rate of evaporation appears to be enhanced relative to a conventional LSIMS source because the sample is supported directly over the source diffusion pump inlet instead of inside a relatively low conductance source ion volume. Probe bias was optimized at $1.9 \mathrm{~V}$, just under the trap potential of $2 \mathrm{~V}$, and intense signals were observed in the analyzer cell (see Figure 6). Only 25 scans, with a total ionization period of $0.125 \mathrm{~s}$, were required to yield this spectrum. Next, $2.9 \mu \mathrm{mol}$ of histidine was added to the glycerol, and the spectrum shown in Figure 7 was obtained by $\mathrm{co}$-adding 10 scans, representing $0.05 \mathrm{~s}$ total ionization time. An abundant $[\mathrm{M}+\mathrm{H}]^{+}$ion, $m / z$ 156 , is apparent, as is a fragment with $m / z 110$, corresponding to decarboxylation of the amino acid. The signal-to-noise (S/N) ratio of the parent ion peak is 131 , recorded under low-resolution (7500 FWHH), broadband conditions (FWHH $=$ full width at half-height). Low-abundance ions from the glycerol are also apparent. Because of the high source pressures it was difficult to accurately measure the transfer efficiency, but it appeared to be $100 \%$.

Another common matrix is the "magic bullet" for peptides, dithiothreitol-dithioerythritol (DTT-DTE), $5: 1$ by weight. With $10 \mu \mathrm{L}$ of this matrix on the probe tip, pressures were $6 \times 10^{-7}$ and $8 \times 10^{-9}$ torr, respectively. Figure 8 shows the spectrum of the DTT-DTE matrix mixture (200 scans, $1 \mathrm{~s}$ ionization time), and Figure 9 shows the spectrum of $2.9 \mu \mathrm{mol}$ of histidine in DTT-DTE (10 scans, $0.05 \mathrm{~s}$ ionization time). The spectrum is very similar to that in Figure 8 , with the exception of different, low-abundance, matrix ions. The parent ion was recorded with an S/N ratio of 156. The inset shows a high-resolution spectrum of the 




Figure 5. SIMS/FTMS spectrum of polyethylene glycol 1000, recorded in the source cell.

protonated molecular ion of histidine, recorded at a pressure-limited resolution of $160,000 \mathrm{FWHH}$. This is approximately $20 \%$ of the EI resolution specification for an FTMS-2000. The signal does not persist as long when DTT-DTE is used as the matrix as when glycerol is used. Also, the abundance of $\mathrm{Cs}^{+}(\mathrm{m} / z$ 133) grows approximately ten times as quickly with DTT-DTE. However, for each of the spectra recorded from a matrix, the sample was still present when the probe was removed from the vacuum system, and further spectra could have been co-added. Again, the transfer efficiency to the analyzer cell appeared to be $100 \%$ [13].

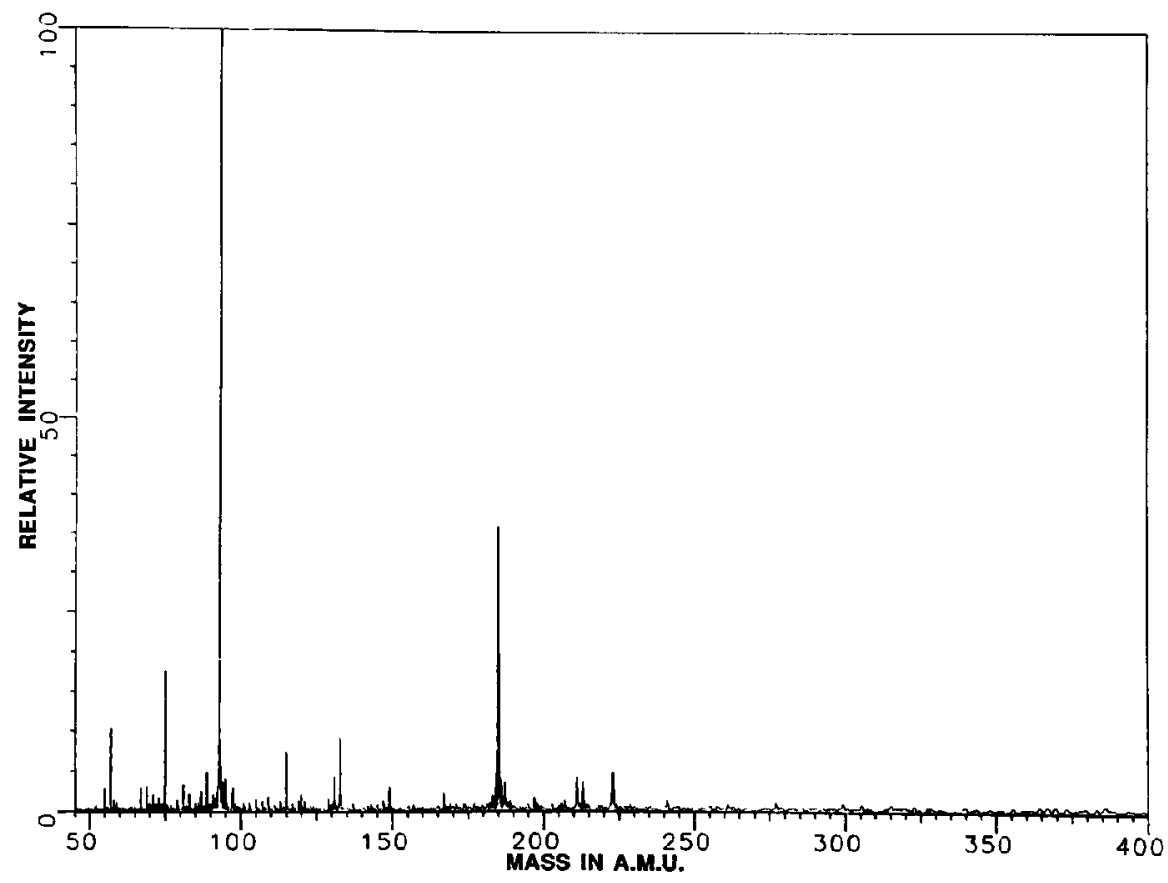

Figure 6. LSIMS/FTMS spectrum of glycerol, recorded in the analyzer cell. 


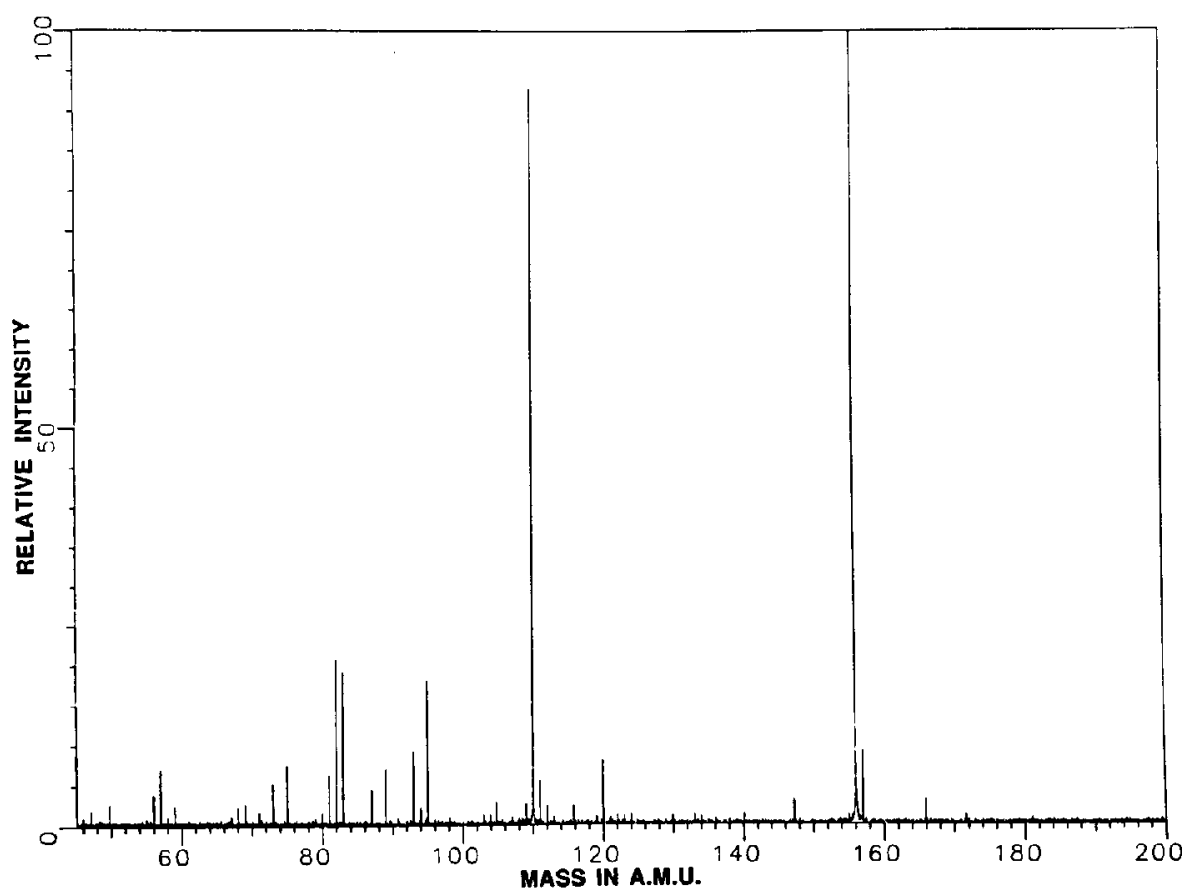

Figure 7. LSIMS/FTMS spectrum of $2.9 \mu \mathrm{mol}$ of histidine in glycerol, recorded in the analyzer cell.



Figure 8. LSIMS/FTMS spectrum of DTT-DTE, 5:1 by weight, recorded in the analyzer cell. 


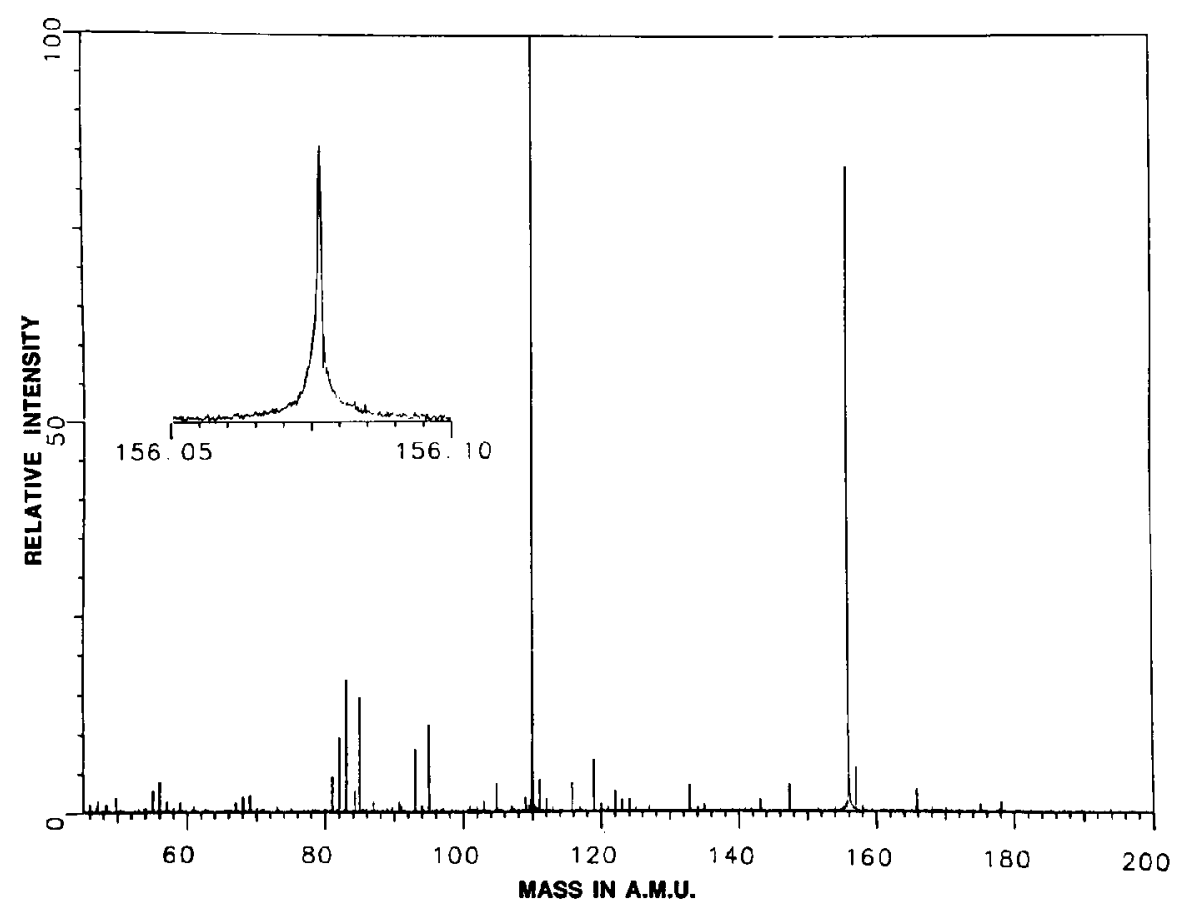

Figure 9. LSIMS/FTMS spectrum of $2.9 \mu \mathrm{mol}$ of histidine dissolved in DTT-DTE, recorded in the analyzer cell. The inset shows the molecular ion recorded separately at a resolution of 160,000 .

\section{Conclusions}

The spectra presented here demonstrate the performance of the external SIMS source. High resolution is possible but is still somewhat pressure-limited when volatile matrices are employed. A standard FTMS-2000 utilizes a $700-\mathrm{L} / \mathrm{s}$ pump for the analyzer region and so has approximately twice the pressure differential obtained in the present instrument. Extension of this approach to the 7.2-T system will require redesign of the cesium ion gun to allow operation transverse to a magnetic field of $0.44 \mathrm{~T}$.

Ion transfer efficiency into the analyzer cell appears to be inversely related to the initial kinetic energy distribution of the secondary ions and, as the trend in probe bias potentials shows, is greatly enhanced by the use of liquid matrices. The larger the initial energy and the more divergent the secondary ion beam, the larger the fraction of $z$-axis velocity converted into cyclotron motion, and the larger the spatial distribution of the ions in the cell. This reduces the fraction that may partition through the $2-\mathrm{mm}$ conductance limit into the analyzer cell, and thus reduces the transfer efficiency. However, this is not a serious limitation, because solid, nonvolatile samples can simply be detected in the source cell at low pressure, and samples dissolved in a liquid matrix can be detected in the analyzer cell.

This paper presents an inexpensive, experimentally simple way to implement an external LSIMS source on a commercially available FTMS, the FTMS-2000, such that all the other capabilities of the instrument are retained. Extra pumps, electrostatic lenses, and radiofrequency quadrupoles are avoided. No attempt has been made yet to maximize the system sensitivity, but these preliminary spectra are quite encouraging.

\section{Acknowledgments}

We gratefully acknowledge support by the National Institutes of Health (GM-30604) and the National Science Foundation (CHE89-11685).

\section{References}

1. Ijames, C. F.; Wilkins, C. L., J. Am. Chem. Soc. 1988, 110 , 2687.

2. Barber, M.; Bordoli, R. S.; Elliot, G. J.; Sedgwick, R. D.; Taylor, A. N. Anal. Chem. 1982, 54, 645A.

3. Aberth, W; Burlingame, A. L. In Ion Formation from Organic Solids; Benninghoven, A., Ed.; Springer-Verlag: Berlin, 1983; pp 167-171.

4. McIver, R. T., Jr.; Hunter, R. L.; Story, M. S.; Syka, J.; Labunsky, M. Proc. 31st ASMS Conf. Mass Spectrom. Allied Topics, Boston, Mass., May 1983; pp 44-45.

5. U.S. Patent $4,535,235$.

6. Hunt, D. F.; Shabanowitz, J.; Yates, J. R., III; Zhu, N.-Z.; Russell, D. H.; Castro, M. E. Proc. Natl. Acad. Sci. U.S.A. 1987, 84, 620.

7. Kofel, P.; Allemann, M.; Kellerhals, H.; Wanczek, K. P. Int. J. Mass. Spectrom. Ion Processes 1985, 65, 97. 
8. Kofel, P.; Allemann, M.; Kellerhals, H.; Wanczek, K. P. Int. J. Mass Spectrom. Ion Processes 1986, 72, 53.

9. Alford, J. M.; Williams, P. E.; Trevor, D. J.; Smalley, R. E. Int. J. Mass Spectrom. Ion. Processes 1986, 72, 33.

10. Lebrilla, C. B.; Amster, I. J.; McIver, R. T., Jr. Int. J. Mass Spectrom. Ion Processes 1989, 87, R7.
11. Castro, M. E.; Russell, D. H. Anal. Chem. 1984, 56, 578.

12. Amster, I. J.; McLafferty, F. W.; Castro, M. E.; Russell, D. H.; Cody, R. B., Jr.; Ghaderi, S. Anal. Chem. 1986, 58, 485.

13. ljames, C. F.; Wilkins, C. L. Proc. 37th ASMS Conf. Mass Spectrom. Allied Topics, Miami Beach, FL, May 1989; pp 1248-1249. 